\title{
Enhancing bone healing in calvarial critical size defect using ozone gel : Histological and histomorphometric analysis
}

\author{
Mohamed Ahmed Elsholkamy', Maggie Ahmed Khairy', Tamer Ahmed Nasr ${ }^{3}$ \\ ${ }^{1}$ Assistant Professor of Oral and Maxillofacial Surgery, Faculty of Dentistry, Suez Canal \\ University, Ismailia, Egypt. \\ ${ }^{2}$ Assistant Professor of Oral and Maxillofacial Surgery, Faculty of Dentistry, October 6 \\ University, Cairo, Egypt. \\ ${ }^{3}$ Lecturer of Oral and Maxillofacial Surgery, Faculty of Dentistry, Misr International \\ University, Cairo, Egypt.
}

\begin{abstract}
Aim: The aim of this study was to examine the validity of the hypothesis that Ozone gel will accelerate bone healing in a critical size defect model experimentally created in rabbit calvaria when mixed with autografts in comparison to autogenous grafts per se.

Materials and Methods: A total of twelve adult male New Zealand Rabbits were included in the study. A total of 24 standardized bone grafts were harvested from 12 animals after critical size defects were created in calvaria cortical bone, each graft was crushed using a special bone mill device. After bone milling, each bone graft was collected in a special sterile container, twelve grafts were mixed with normal saline solution (control group) and each one of the rest of the grafts was mixed with ozone gel. Animals were sacrificed at 4 and 8 weeks' post-surgery, dual energy x-ray absorptiometry (DEXA) scans were performed for the skull of the rabbits and bone specimens were collected for histological examination. Results: Histomorphometric analysis showed superior results in favor of the ozone treated group represented as a significantly higher percentage of normal osteocytes and marked increase in area percentage of new bone formation. Additionally, DEXA scan revealed a significant increase in bone mineral density and bone mineral concentration of the ozone treated group compared to the control group.

Conclusion: The authors believe that according to the available results the use of ozone gel may be cost effective and convenient owing to its ease of preparation. It is recommended to be used with routine bone grafting procedures as it accelerates the new bone formation over time giving higher degree of overall maturation and strength.
\end{abstract}

Key Words: Autogenous bone grafts, calvarial, critical size defect, ozone gel, rabbit model.

Received: 19 June 2018, Accepted: 29 July 2018

Corresponding Author: Nasr, Tamer, Department of Oral and Maxillofacial Surgery, Faculty of Dentistry, Misr International University, Cairo, Egypt, Tel.: 0100157959, E-mail: tamer.nasr129@gmail.com.

ISSN: 2090-097X, May 2018, Vol. 9, No.2

\section{INTRODUCTION}

The complete remodeling process of bone graft and regaining of bone form and strength was the goal of multitude of research literature ${ }^{[1-3]}$. Undisturbed normal bone healing process with the aid of bone grafting material either autogenous or synthetic materials gained large attention in the past decades ${ }^{[4-9]}$.

Many materials showed different degrees of success rates and different related complications. This makes autogenous bone the supreme type for grafting in spite of the donor site morbidity but surgically augmented height with an autogenous block graft decreased to $60 \%$ after 10 months ${ }^{[10]}$.
It is a hallmark standard among researchers for testing new bone substitutes in calvarial defects of the rat and rabbit, followed by testing in the mandibles of dogs and monkeys using a critical size defect (CSD) which is the diameter of a bone wound such that beyond that amount complete calcification of the wound will not occur during the lifetime of the anima ${ }^{[11]}$.

Among recent bone enhancing techniques Ozone therapy gained access to the field of dentistry in many approaches ${ }^{[12-18]}$, there is insufficient evidence in the application of ozone in oral and maxillofacial surgery ${ }^{[19]}$. As ozone has a therapeutic effect that facilitates wound healing and improves the supply of blood, ozone therapy could enhance the stability and predictability of autogenous grafts. 
The aim of this study was to examine the validity of the hypothesis that Ozone gel will accelerate bone healing in a critical size defect model experimentally created in rabbit calvaria when mixed with autografts in comparison to autogenous grafts per se.

\section{MATERIAL AND METHODS}

This is an experimental controlled study that includes two groups; a control group comprised 12 autogenous calvarial grafts, and a study group comprised 12 autogenous calvarial grafts mixed with ozone gel. The study was conducted following the approval of October 6 Univeristy ethical committee, faculty of dentistry (Approval number 2017/3) for animal use. A total of twelve adult male New Zealand Rabbits (Oryctolagus cuniculus) were included in the study with an average weight of $3.5-4 \mathrm{~kg}$., age 6 to 8 -months-old. Animals were kept in individual cages in a standard day / night cycle of 12 hours. They were allowed free access to water and laboratory food. The rabbits were randomly divided into two equal groups as follows:

- Control group $(\mathrm{n}=12)$, receiving only autograft without ozone therapy. (Group I). Test group $(\mathrm{n}=12)$, receiving graft mixed with ozone gel (Group II)

\subsection{Ozone gel preparation.}

The ozone gel was obtained by bubbling of $25 \mu$ / $\mathrm{ml} \mathrm{O} 3$ gas through pure olive oil for 2 days until olive oil transforms from greenish colored liquid status to the whitish gel status. This procedure was performed by the longevity Ext 120 ozone generator. (Longevity Extra120, Longevity Co., Canada)

\subsection{Surgical technique}

The animals were premedicated using midazolam $(0.2 \mathrm{mg} / \mathrm{kg})$. General anesthesia was induced by intramuscular injection of ketamine $(10 \mathrm{mg} / \mathrm{kg}$ of body weight), $2 \%$ xylazine $(4 \mathrm{mg} / \mathrm{kg}), 0.2 \%$ acepromazine $(0.15 \mathrm{mg} / \mathrm{kg})$ and an intravenous propofol $(2 \mathrm{mg} / \mathrm{kg})$ Local anesthesia (mepivicaine 2\% containing 1:100.000 levonordephrine) was infiltrated around the surgical site. Prophylactic enrofloxacin antibiotics were administrated via intravenous route $(5 \mathrm{mg} / \mathrm{kg}$ of body weight). The skin at the operative site was shaved and scrubbed using $2 \%$ iodine solution. A midline incision from the frontal area to the occipital protuberance was made down to the osseous surface of the skull, and a full thickness flap was raised to expose the calvarial surface on both sides of the midline. (Fig. 1)

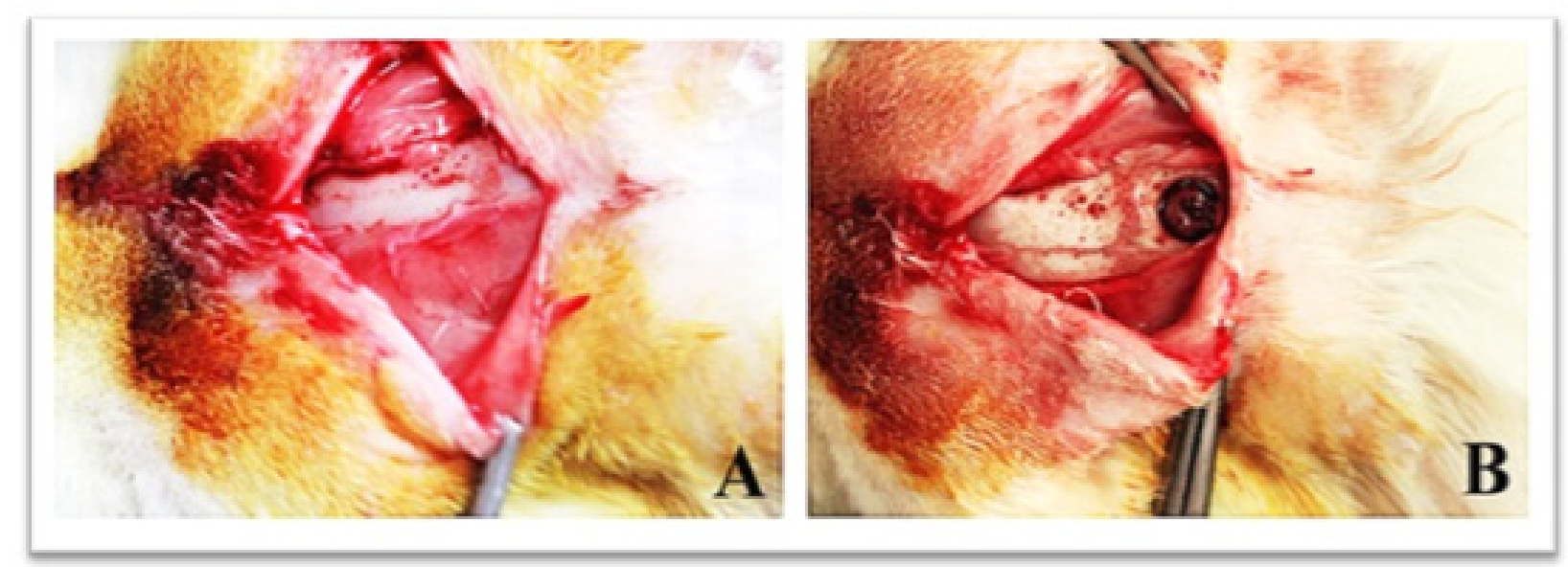

Fig. 1: Photographs showing the midline calvarial incision (A), the calvarial defect after taking the graft (B).

Attention was made to avoid perforation of the underlying dura mater and not to involve the sagittal suture. A standardized bone graft was obtained from each animal using a trephine bur with an inner diameter of $5 \mathrm{~mm}$ mounted on a hand piece at 2,000 rpm under copious saline solution irrigation. The graft consisted of both the outer and inner calvaria cortical bone, which was approximately $3 \mathrm{~mm}$ thick with a diameter of $5 \mathrm{~mm}$. A total of 24 grafts were harvested from 12 animals, each graft was crushed using a special bone mill device (Fig 2).

After bone milling, each bone graft was collected in a special sterile container. After preparing the recipient site, bone graft that was grounded with a manual bone crushed mixed with normal saline, and was implanted in the bone defect of Group I. In Group II same procedure was done and bone graft was mixed by Ozone gel and implanted in the bony defect saline solution 


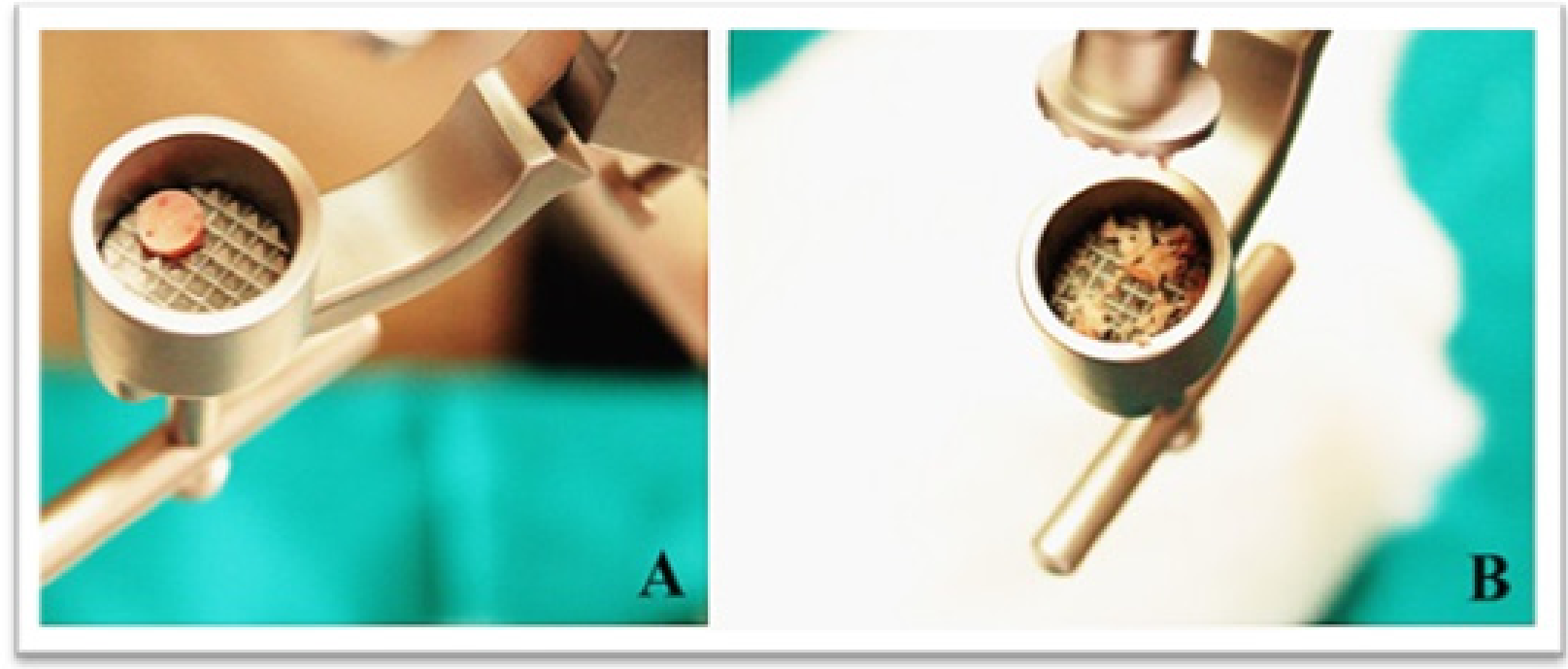

Fig. 2: Photographs showing (A) The graft within the bone mill, (B) The graft after milling.

The soft tissues were then repositioned and sutured to achieve primary closure (4-0 silk). To prevent postoperative infection, ceftriaxone was given to the animals as intramuscular injections for $3 \mathrm{~d}(30 \mathrm{mg} / \mathrm{kg})$. They were also given an intramuscular analgesic, $4 \mathrm{mg} / \mathrm{kg}$ carprofen every $24 \mathrm{~h}$ for 3 days, starting immediately after the operation.

\section{3. Histological technique}

Upon completion of the experimental periods for each group, the animals were euthanized by over dose anesthesia, and the area of the original surgical defect and the surrounding tissues were removed en bloc and fixed in $10 \%$ buffered formalin for $48 \mathrm{~h}$ hours. The specimens were then decalcified in $20 \%$ formic acid and $10 \%$ sodium citrate for 10 days, cut transversely next to the hole, and embedded in paraffin according to standard histological procedures. Five micrometer thick serial sections were cut, stained with hematoxylin and eosin to be evaluated under a light microscope by a single oral pathologist. (Blind Evaluation)

The best sections (which were given codes) were used for evaluation for osteoblastic activity, new bone formation or any inflammatory reactions. The Pathologist's observations were tabulated and then the codes were revealed by the authors.

Leica application suite (LAS V4) system (Switzerland) and Image $\mathrm{J}$ image analysis software were used to lock on these preselected areas for each histological section. For each of the two studied groups a differential osteocyte count (normal osteocyte, abnormal osteocyte, empty lacunae) was performed for each section. The osteocytes were classified according to the morphological criteria established by Moura et al. ${ }^{[20]}$, those that occupied more than $50 \%$ of their lacunae were considered normal, and those that occupied $50 \%$ or less of their lacunae were considered abnormal. Empty lacunae were also counted.

The sequence of bone repair was observed histologically by examining the two groups at either 7 or 14 days by manually counting the area percentage of new bone and expressing it as areas (in $\mathrm{mm} 2$ ). To standardize our histomorphometric analysis, we based our measurements in part on the work of Messora et al. ${ }^{[21]}$, The total area (TA) to be analyzed corresponded to the entire area of the original surgical defect. The mineral deposition area (MDA) was delineated within the confines of the TA. The TA was measured in $\mathrm{mm} 2$ and was considered $100 \%$ of the area to be analyzed. The MDA was also measured in $\mathrm{mm} 2$ and calculated as a percentage of TA. The number of inflammatory cell infiltrate to the marrow spaces were also recorded for each histological section obtained in the two-time intervals.

\section{4. Bone densitometry}

The skulls of the animals were harvested, after animal scarification, and a dual energy x-ray absorptiometry (DEXA) scan was performed for each skull using a peripheral DEXA device. The area of interest was detected, centralized and scanned using an examination surface area of $4 \pm 0.5 \mathrm{~cm} 2$. Bone mineral density (BMD module was verified and represented. The data for each group at 7 and 14 days were recorded in table for statistical analysis.

\section{RESULTS}

\subsection{Animal recovery}

Healing progressed uneventfully in all animals and no postoperative complications were noticed. 


\subsection{Descriptive histology}

Histologically, bone matrix was secreted at day 7 and increased significantly at day 14. Osteoblastic cells appeared at the early stages of 7 days and matured over time. Osteogenic activity was detected directly at the interface. A higher degree of formation of vascularized tissues, of provisional matrix, and of bone remodeling activity at 7 and 14 days was recorded in the ozone group as compared to the control group. Meanwhile, a higher number of normal osteocytes were detected in the ozone group.

At 7 days: Bone formation did not occur at a uniform

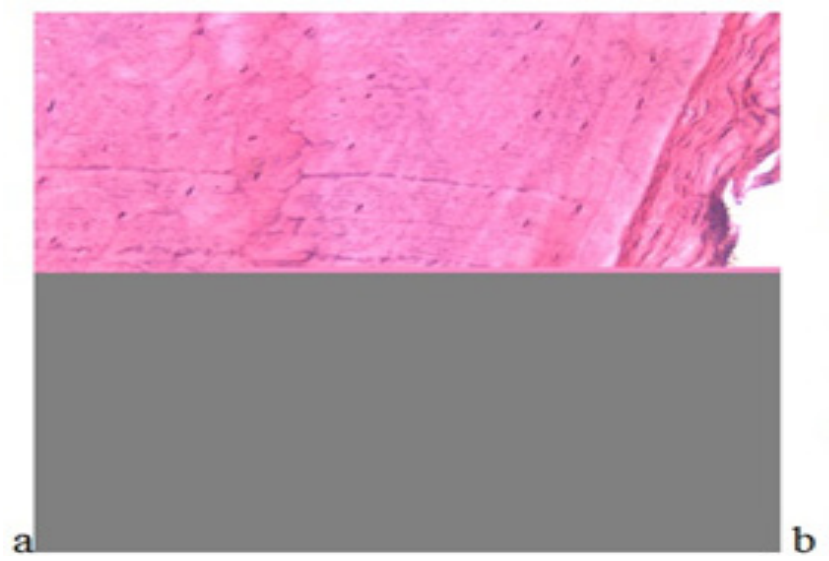

rate across the entire defect. The initial synthesis of bone repair at 7 days was woven and confined adjacent to the preexisting lamellar cortical bone. Initially, new bone synthesis occurred peripherally restricted to areas that were close to the borders of the surgical defect. The biggest central part of the surgical defect was occupied by connective tissue with collagen fibers parallel to the wound surface with a mild to moderate chronic inflammatory infiltrate. The complete closure of the defect was not observed at day 7 . The repair process is associated with a rich vascular front, which primarily forms from the marrow and passages into the defect at right angles to the long axis of the bone (Figure 3 ).

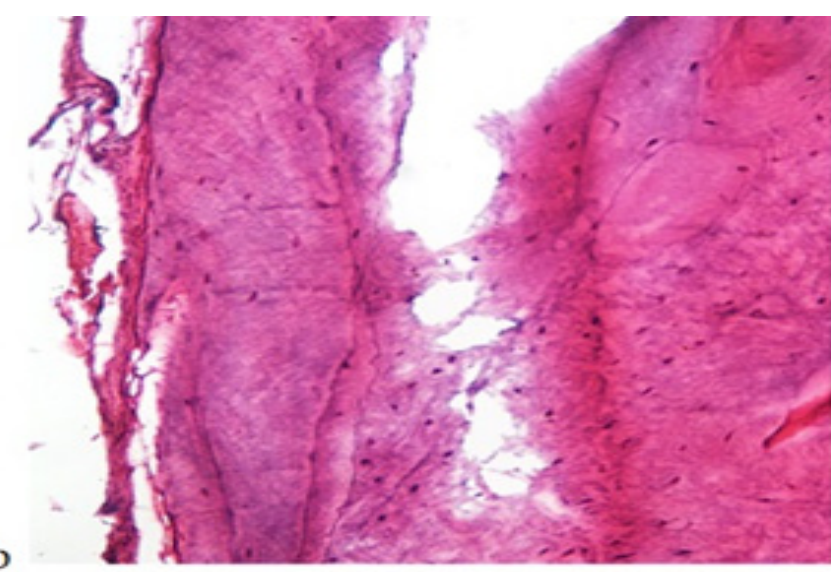

Fig. 3: Photomicrographs at 7 days $\left({\left.\mathrm{H} \& \mathrm{E}^{\circ}-200\right)}^{\circ}\right.$ (A) Control group showing the parallel arrangement of the collagen fibers in relation to the surface of the surgical defect, the collagen was restricted to areas that were close to the borders of the surgical defect while the center of the defect was still empty. (B) Ozone group showing woven bone attempting to close the defect. Note that the newly formed bone was mainly restricted to areas close to the borders of the defect (top) although attempts of bridging were seen at the bottom of the photomicrograph.

Postoperative results at 7 and 14 days: All bone defects in the two groups healed with full regeneration of bone. The ozone group showed considerable faster healing at the end of the 14 days' period together with decreased number of inflammatory cells. Bone at the periphery, which was originally woven was transformed into lamellar bone adjacent to the persisting cortices. Closer towards the center of the defect woven bone predominated. All the defects of both groups were mainly filled by newly formed woven bone with thin and irregular trabeculae surrounded by fibro-vascular tissue. The woven bone was rimmed by plump surface osteoblasts. The ozone group was bridged by mineralized bone with irregular shape and volume (Figure 4).
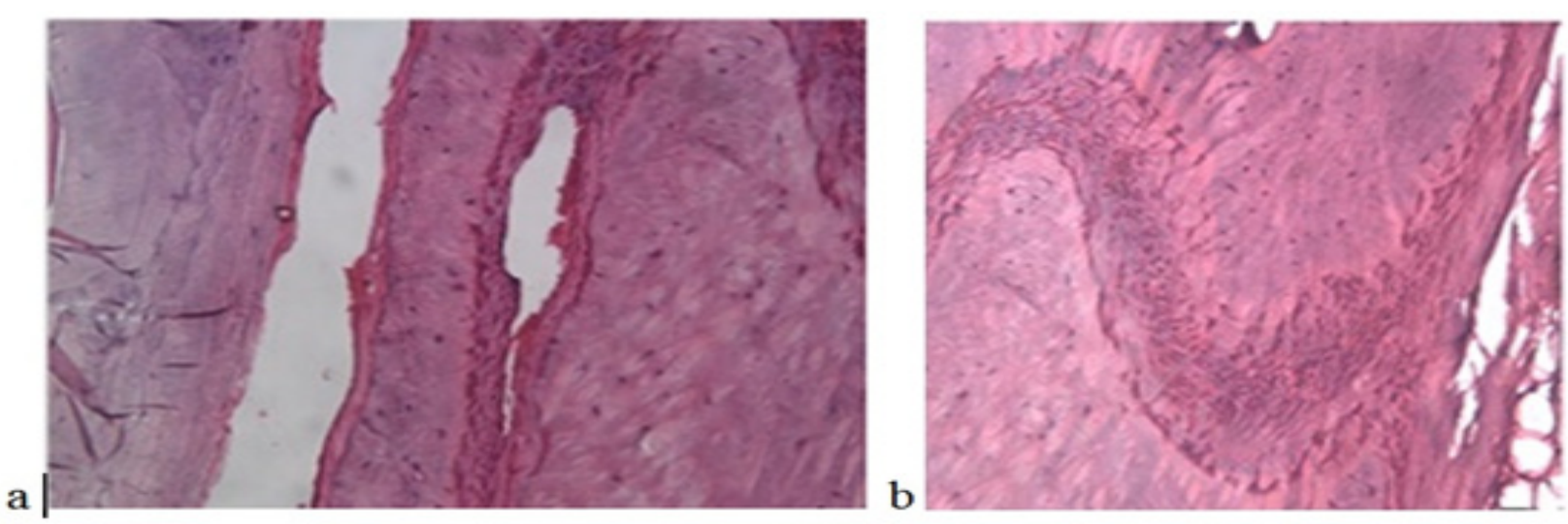

Fig. 4: Photomicrographs at 14 days ( $\left.\mathrm{H} \& \mathrm{E}^{\circ}-200\right)$ : (A) Control group showing the longitudinal orientation of repair tissue (B) Ozone group showing complete closure of the surgical defect by mineralized bone trabeculae in a fibro-vascular stroma 


\subsection{Statistical Analysis}

Numerical data were explored for normality by checking the distribution of data and using tests of normality (Kolmogorov-Smirnov and Shapiro-Wilk tests). All data showed normal (parametric) distribution except for number of inflammatory cells data which showed nonnormal (non-parametric) distribution. Data were presented as mean and standard deviation (SD) values.

For parametric data, two-way analysis of Variance (ANOVA) was used to study the effect of group and time on different variables. Bonferroni's post-hoc test was used for pair-wise comparisons when ANOVA test is significant. For non-parametric data, Mann-Whitney U test was used to compare between the two groups as well as to compare between the two follow-up times.

The significance level was set at $P \leq 0.05$. Statistical analysis was performed with IBM SPSS Statistics Version 20 for Windows.

\subsubsection{Statistical Analysis Outcomes 3.3.1.1 Percentage of normal osteocytes}

After 7 as well as 14 days; Ozone gel group showed statistically significant higher mean percentage of normal osteocytes than control group. In both groups, the mean percentage of normal osteocytes after 14 days showed statistically significantly higher mean value than after 7 days (Table 1) (Figure 5).

Table 1: The mean, standard deviation (SD) values and results of two-way ANOVA test for comparison between percentage of normal osteocytes in the two groups as well as the change by time within each group

\begin{tabular}{|c|c|c|c|c|c|c|}
\hline & \multicolumn{2}{|c|}{ Ozone gel } & \multicolumn{2}{|c|}{ Control } & \multirow{2}{*}{$\begin{array}{l}P \text {-value (Between } \\
\text { groups) }\end{array}$} & \multirow{2}{*}{ Effect size } \\
\hline & Mean & $\mathrm{SD}$ & Mean & $\mathrm{SD}$ & & \\
\hline 7 days & 30.2 & 3.3 & 23.8 & 3.1 & $<0.001 *$ & 0.512 \\
\hline 14 days & 40.7 & 4.8 & 36.6 & 3.2 & $0.024 *$ & 0.211 \\
\hline$P$-value (Within group) & \multicolumn{2}{|c|}{$<0.001 *$} & \multicolumn{2}{|c|}{$<0.001^{*}$} & & \\
\hline Effect size & \multicolumn{2}{|c|}{0.784} & \multicolumn{2}{|c|}{0.842} & & \\
\hline
\end{tabular}

*: Significant at $P \leq 0.05$

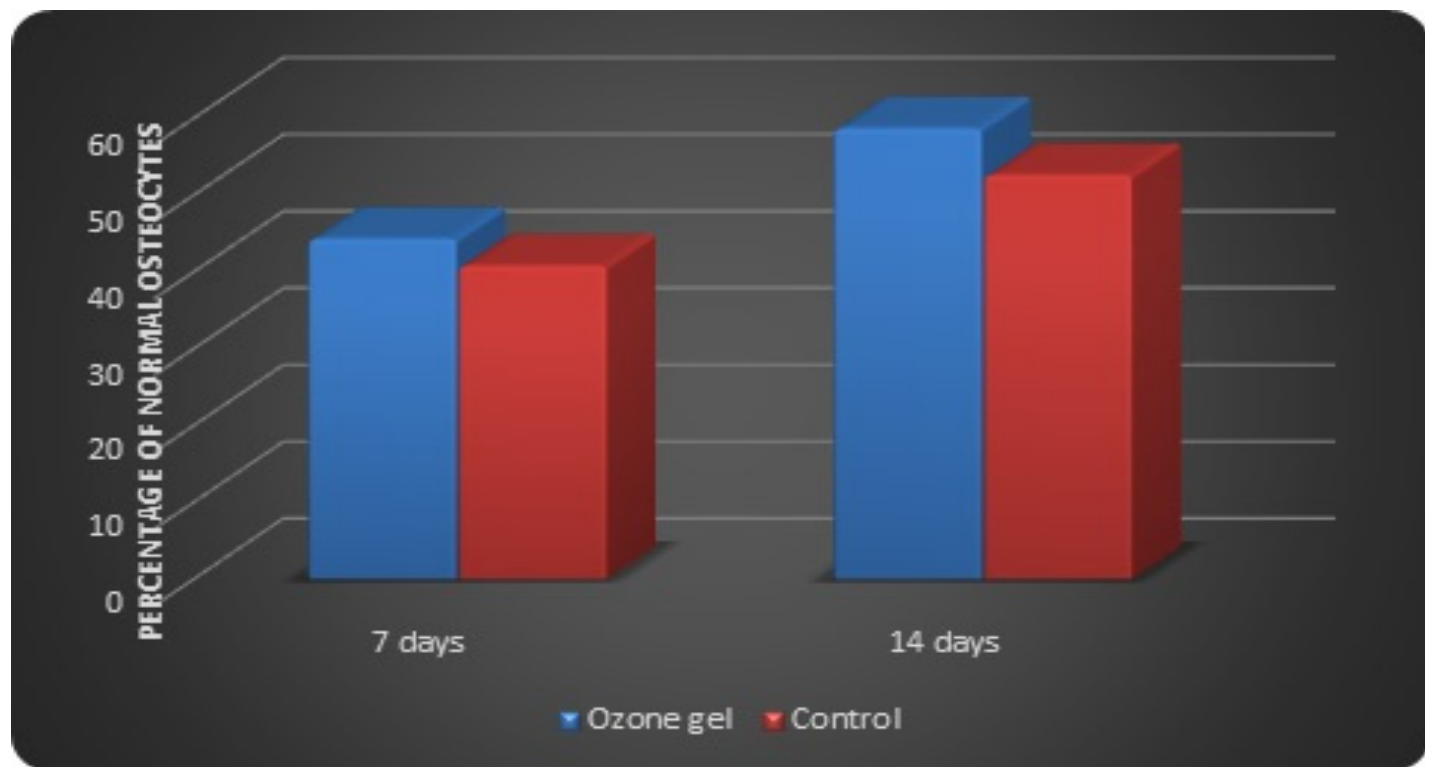

Fig. 5: Bar chart representing mean normal osteocytes percentage in the two groups 


\subsubsection{Area percentage of new bone}

After 7 as well as 14 days; Ozone gel group showed statistically significant higher mean area percentage of new bone than control group. In both groups, the mean area percentage of new bone after 14 days showed statistically significant higher mean value than after 7 days (Table 2) (Figure 6).

Table 2: The mean, standard deviation (SD) values and results of two-way ANOVA test for comparison between area percentage of new bone in the two groups as well as the change by time within each group

\begin{tabular}{|c|c|c|c|c|c|c|}
\hline & \multicolumn{2}{|c|}{ Ozone gel } & \multicolumn{2}{|c|}{ Control } & \multirow{2}{*}{$\begin{array}{l}P \text {-value (Between } \\
\text { groups) }\end{array}$} & \multirow{2}{*}{ Effect size } \\
\hline & Mean & SD & Mean & SD & & \\
\hline 7 days & 44.3 & 3.8 & 40.8 & 4.6 & $0.046^{*}$ & 0.156 \\
\hline 14 days & 58.7 & 3.9 & 52.6 & 5.7 & $0.006^{*}$ & 0.295 \\
\hline$P$-value (Within group) & \multicolumn{2}{|c|}{$<0.001 *$} & \multicolumn{2}{|c|}{$<0.001 *$} & & \\
\hline Effect size & \multicolumn{2}{|c|}{0.904} & \multicolumn{2}{|c|}{0.863} & & \\
\hline
\end{tabular}

*: Significant at $\mathrm{P} \leq 0.05$

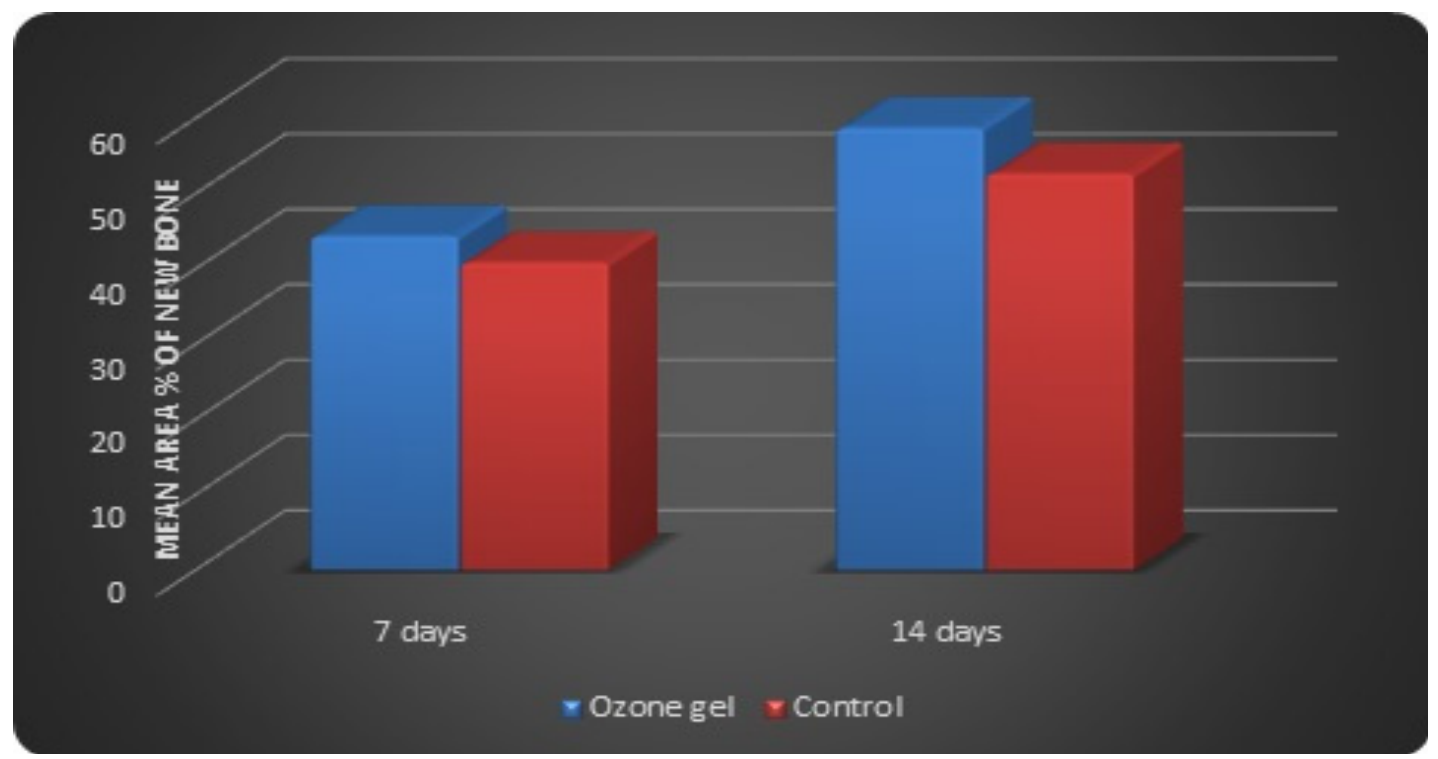

Fig. 6: Bar chart representing mean area percentage of new bone in the two groups

\subsubsection{Number of inflammatory cells}

After 7 as well as 14 days; Ozone gel group showed statistically significant lower mean between number of inflammatory cells than control group. In both groups, the mean number of inflammatory cells after 14 days showed statistically significant lower mean value than after 7 days (Table 3) (Figure 7). 
Table 3: The mean, standard deviation (SD) values and results of Mann-Whitney $U$ test for comparison between number of inflammatory cells in the two groups as well as the change by time within each group

\begin{tabular}{|c|c|c|c|c|c|c|}
\hline & \multicolumn{2}{|c|}{ Ozone gel } & \multicolumn{2}{|c|}{ Control } & \multirow{2}{*}{$\begin{array}{l}P \text {-value (Between } \\
\text { groups) }\end{array}$} & \multirow{2}{*}{ Effect size } \\
\hline & Mean & SD & Mean & SD & & \\
\hline 7 days & 10.6 & 3.8 & 15.1 & 4.0 & $0.046^{*}$ & 0.156 \\
\hline 14 days & 5.2 & 2.7 & 9.0 & 1.9 & $0.006^{*}$ & 0.295 \\
\hline$P$-value (Within group) & \multicolumn{2}{|c|}{$<0.001 *$} & \multicolumn{2}{|c|}{$<0.001^{*}$} & & \\
\hline Effect size & \multicolumn{2}{|c|}{0.904} & \multicolumn{2}{|c|}{0.863} & & \\
\hline
\end{tabular}

*: Significant at $P \leq 0.05$

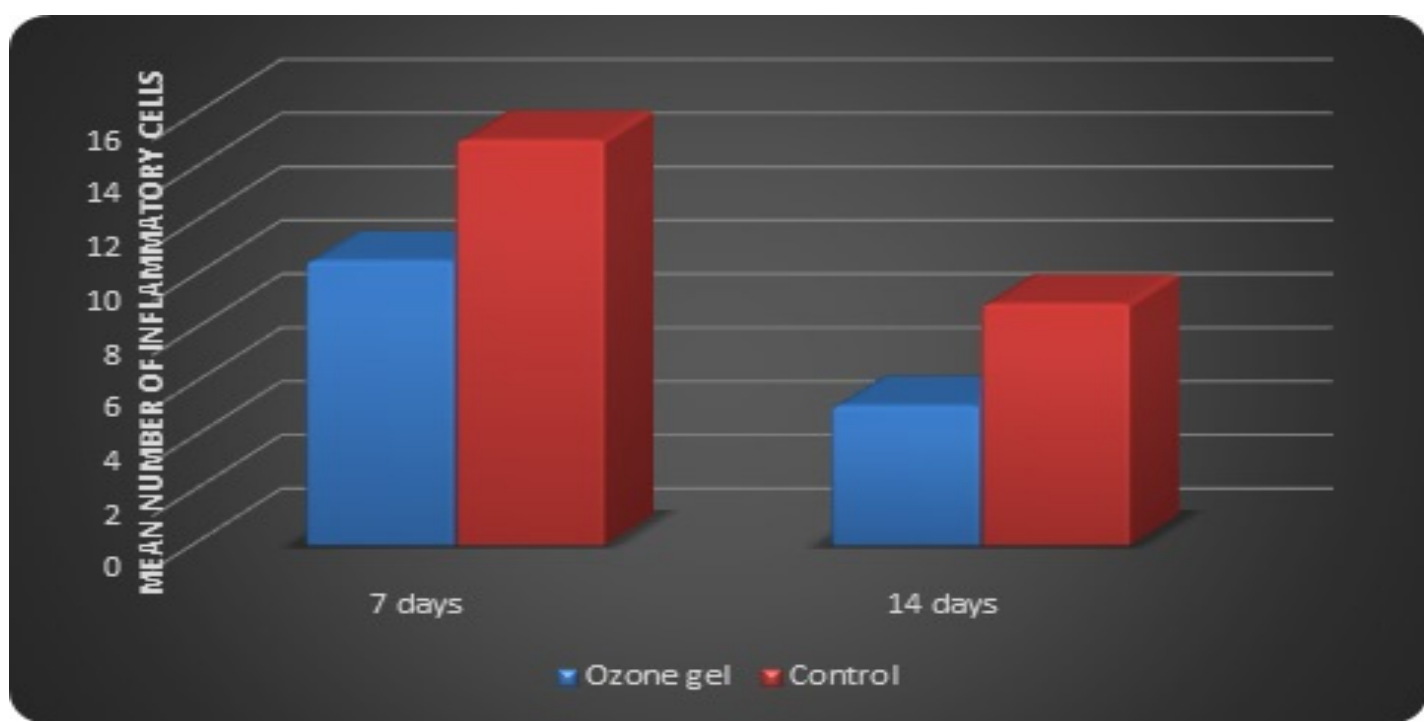

Fig. 7: Bar chart representing mean number of inflammatory cells in the two groups

\subsubsection{Bone Mineral Density (BMD)}

After 7 as well as 14 days; there was no statistically significant difference between bone mineral density in the

two groups. However, in both groups, the mean area BMD after 14 days showed statistical significant higher mean value than after 7 days (Table 4) (Figure 8).

Table 4: The mean, standard deviation (SD) values and results of two-way ANOVA test for comparison between Bone Mineral Density (BMD) in the two groups as well as the change by time within each group

\begin{tabular}{|c|c|c|c|c|c|c|}
\hline & \multicolumn{2}{|c|}{ Ozone gel } & \multicolumn{2}{|c|}{ Control } & \multirow{2}{*}{$\begin{array}{l}P \text {-value (Between } \\
\text { groups) }\end{array}$} & \multirow{2}{*}{ Effect size } \\
\hline & Mean & SD & Mean & SD & & \\
\hline 7 days & 0.28 & 0.05 & 0.26 & 0.04 & 0.359 & 0.033 \\
\hline 14 days & 0.58 & 0.07 & 0.52 & 0.06 & 0.069 & 0.143 \\
\hline$P$-value (Within group) & \multicolumn{2}{|c|}{$<0.001^{*}$} & \multicolumn{2}{|c|}{$<0.001 *$} & & \\
\hline Effect size & \multicolumn{2}{|c|}{0917} & \multicolumn{2}{|c|}{0.896} & & \\
\hline
\end{tabular}

*: Significant at $P \leq 0.05$ 


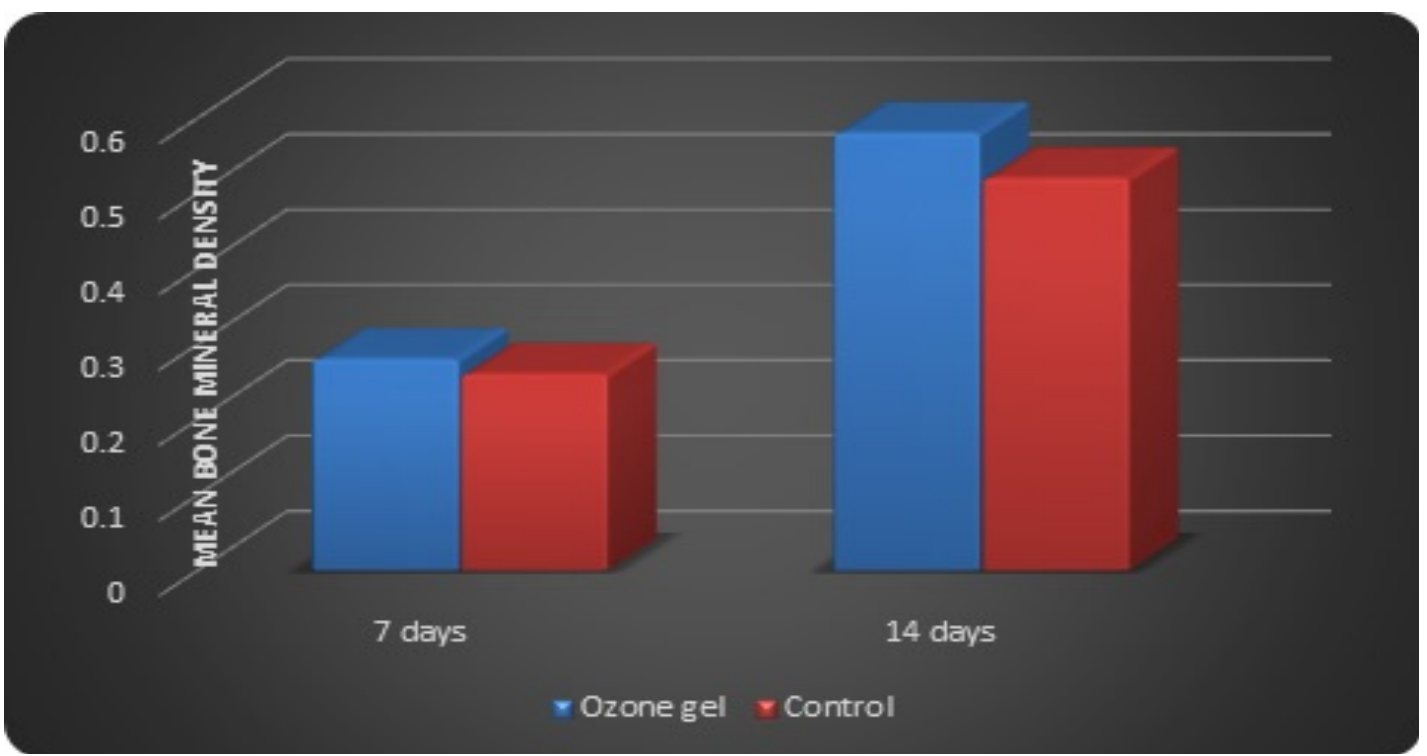

Fig. 8: Bar chart representing mean Bone Mineral Density (BMD) in the two groups

\section{DISCUSSION}

Bone healing and regeneration to normal form and function is the ultimate goal after surgical procedures involving intra-bony pathological lesions surgical management, this goal was for decades an interesting point of both experimental and clinical research, utilizing of many materials for achieving this goal with different degree of success.

Several treatment modalities were recently used, such as low-level laser therapy which was effective for stimulating bone formation in critical size defects in the calvaria of rats submitted to ovariectomy, the results were in agreement with our results. Hyperbaric oxygen therapy was also used in enhancing bone healing in ungrafted rabbit calvarial critical-sized defects and may likewise increase the rate of residual graft resorption in autogenous bone-grafted defects ${ }^{[22-26]}$. However, the use of low-level laser therapy requires expensive equipment with hazards for both the patient and the operator, also hyperbaric oxygen therapy is time consuming and utilizes chamber with patient commitment to attend many dives.

The rabbit model was used for this study because the bone repair process of rabbits, although faster, is physiologically similar to that of humans ${ }^{[27,28]}$. Furthermore, the use of cranial bone for grafting purposes has some significant benefits, such as a higher amount of surviving bone graft and a relatively short postoperative recovery period. Moreover, cranial bone harvesting is a relatively safe procedure, with a low morbidity compared to iliac crest bone harvesting ${ }^{[29]}$. Additionally, and most emphasized feature, is the embryologic, morphologic, and physiologic similarity of this bone to that in the maxillofacial region ${ }^{[30]}$.

Bocci stressed that during ozone therapy, ozone triggers a series of biological mechanisms that lead to normalizing the delivery of oxygen for several days with consequent therapeutic effects ${ }^{[12]}$. Ozone delivery system can provoke several responses on the biological aspect of bone regeneration, such as improvement of the blood circulation in ischemic tissue by increasing oxygen delivery and enhancement of the general metabolism via mild activation of the immune system and upregulation of cellular antioxidant enzymes and growth factors ${ }^{[31]}$.

Unfortunately, few literature has utilized Ozone in calvarial bone defects, the histological findings of the current study revealed an obvious enhancement in new bone formation and a marked reduction in concentration of inflammatory cells of the ozone gel treated specimens. These results are in accordance with Ozdemir et al. who found similar results of increase bone healing when utilized in calvaria of rats in terms of increase osteoblast number and new bone formation these observations showed superior results for ozone therapy group, both in histomorphometric assessments (using image analysis software), and histological analyses, compairing ozone therapy to controlled non-grafted group and autogenous graft without ozone therapy ${ }^{[32]}$.

Ozdemir et al. used sophisticated technology using Ozonix Ozone Generator, a device that produces ozone at a fixed concentration through a connected hand-piece. The use of this device is an additional cost and requires skillful operator.

In our study, Bone densitometry measurements and area percentage of new bone showed an understandable increase in mean values within the ozone gel group, however mineral bone density and concentration mean value increase was not statistically significant. This may be considered as an additional confirmatory verdict to 
the distinct biocompatibility of ozone gel as a potential additional benefit for bone grafts as it increases the new bone formation thus help accelerating bone filling and maturation rates in comparison to control group.

\section{DISCUSSION}

The authors believe that according to the available results the use of ozone gel may be cost effective and convenient owing to its ease of preparation. It is recommended to be used with routine bone grafting procedures as it accelerates the new bone formation over time giving higher degree of overall maturation and strength.

\section{ACKNOWLEDGEMENT}

The authors would like to express their deep gratitude to Prof. Heba Dahmoosh, Oral Pathology Dept., Faculty of Dentistry, Cairo University.

\section{CONFLICT OF INTEREST}

There are no conflicts of interest.

\section{REFERENCES}

1. Wenhao W., Kelvin W.K. Yeung Bone grafts and biomaterials substitutes for bone defect repair: A review. Bioactive Materials 2 (2017) 224-247.

2. Masahiro Y., Hiroshi E. Current bone substitutes for implant dentistry. Journal of prosthodontic research 62 (2018) 152-161.

3. Ibrahim E., Alberto T., Wei X., Birgitta N., Anna J., Christer D., Peter T. and Omar O. Guided bone regeneration using resorbable membrane and different bone substitutes: Early histological and molecular events., Acta Biomaterialia 29 (2016) 409-423.

4. Galo F. G. C., Mayra E. P. M., Juan A. B. B.,Katerine I. N. B., and Denisse V. S. G. Gingival and bone tissue healing in lower third molar surgeries. Comparative study between use of platelet rich fibrin versus physiological healing.. Revista Odontológica Mexicana, Vol. 21, (2017) No. 2 April-June.

5. Jung-Seok L., Seul K. K., Byung-Joo J., SeongBok C., Eun-Young C. and Chang-Sung K. Enhancing proliferation and optimizing the culture condition for human bone marrow stromal cells using hypoxia and fibroblast growth factor-2, Stem Cell Research (2018) 28 :87-95.
6. Cecchi, Simon J B. and Manit A. Bone morphogenetic protein-7: Review of signalling and efficacy in fracture healing Steven Journal of Orthopaedic Translation (2016) 4: 28-34

7. Healing of a Large Long-Bone Defect through Serum-Free In Vitro Priming of Human Periosteum-Derived Cells. Stem Cell Reports, Vol. 8, (2017) 758-772: March.

8. Joensuua K., Uusitaloa L., Almb J.J., Arob H.T., Hentunena T.A. and Heinoa T.J. Enhanced osteoblastic differentiation and bone formation in co-culture of human bone marrow mesenchymal stromal cells and peripheral blood mononuclear cells with exogenous VEGF., Orthopaedics and Traumatology: Surgery and Research 101 (2015) 381-386.

9. Elcin B., Selim E. and Volkan A., Vascular endothelial growth factor and biphasic calcium phosphate in the endosseous healing of femoral defects: An experimental rat study. Journal of Dental Sciences 12, (2017) 7-13.

10. Oh K.C., Cha J.K., Kim C.S., Choi S.H., Chai J.K. and Jung U.W. The influence of perforating the autogenous block bone and the recipient bed in dogs. Part I: a radiographic analysis. Clin Oral Implants Res (2011); 22: 1298-1302.

11. El-Rashidy A. A., Judith A. R., Leila H., Ulrich K. and Aldo R. B. Regenerating bone with bioactive glass scaffolds: A review of in vivo studies in bone defect models. Acta Biomaterialia 62 (2017) 1-28.

12. Bocci V.A., Scientific and medical aspects of ozone therapy. State of the art. Arch Med Res 2006; 37:425-435.

13. Sagai M. and Bocci V. Mechanisms of action involved in ozone therapy: Is healing induced via a mild oxidative stress? Med Gas Res 2011; 20: 1-29.

14. Polydorou O., Halili A., Wittmer A., Pelz K. and Hahn P. The antibacterial effect of gas ozone after 2 months of in vitro evaluation. Clin Oral Investig 2012; 16: 545-550.

15. Huth K.C., Jakob F.M., Saugel B. et al. Effect of ozone on oral cells compared with established antimicrobials. Eur J Oral Sci 2006; 114: 435-440.

16. Polydorou O., Pelz K. and Hahn P. Antibacterial effect of an ozone device and its comparison with two dentin-bonding systems. Eur J Oral Sci 2006; 114: 349-353. 
17. Baysan A. and Lynch E. Effect of ozone on the oral microbiota and clinical severity of primary root caries. Am J Dent 2004; 17: 56-60.

18. Azarpazhooh A. and Limeback H. The application of ozone in dentistry: a systematic review of literature.. J Dent 2008; 36: 104-116.

19. Azarpazhooh A. and Limeback H. The application of ozone in dentistry: a systematic review of literature. J Dent 2008; 36: 104-116.

20. Moura C.G., Betoni W.J. and Dechichi P. Histomorphometric analysis of bone graft storage in physiologic solution [in Portuguese]. Cienc Odontol Bras. 2005; 8(1): 23-27.

21. Messora M.R., Nagata M.J., Mariano R.C., Dornelles R.C., Bomfim S.R. and Fucini S.E. Bone healing in critical-size defects treated with platelet-rich plasma: a histologic and histometric study in rat calvaria. J Periodontal Res. 2008; 43: 217-223.

22. Jan A., Sandor G.K., Brkovic B.B., Peel S., Evans A.W. and Clokie C.M. Effect of hyperbaric oxygen on grafted and nongrafted calvarial critical-sized defects. Oral Surg Oral Med Oral Pathol Oral Radiol Endod 2009; 107: 157-163.

23. Altundal H. and Gursoy B. The influence of alendronate on bone formation after autogenous free bone grafting in rats. Oral Surg Oral Med Oral Pathol Oral Radiol Endod 2005; 99: 285-291.

24. Garcia V.G., da Conceic $\sim$ ao J.M., Fernandes L.A. et al. Effects of LLLT in combination with bisphosphonate on bone healing in critical size defects: a histological and histometric study in rat calvaria. Lasers Med Sci 2012; doi: $10.1007 /$ s10103-012-1068-5.

25. Juliano M., Ricardo O., David J. R. G., et al.
Influence of low-level laser therapy on the healing process of autogenous bone block grafts in the jaws of systemically nicotine-modified rats: A histomorphometric study, Alvaro Francisco Bosco. Archives of Oral Biology 75 (2017) 21-30.

26. Carolina dos S. S., Hiskell F. F. O., Victor E. de S. B., Cleidiel A. A. L., Fellippo R. V. BMP2 expressing genetically engineered mesenchymal stem cells on composite fibrous scaffolds for enhanced Influence of low-level laser therapy on the healing of human bone maxillofacial defects: A systematic review. Journal of Photochemistry and Photobiology, B: Biology 169 (2017) 83-89.

27. Frame J.W. A conventional animal model for testing bone substitute materials. J Oral Surg 1980; 38: 176- 180 .

28. Schmitz J.P. and Hollinger J.O. The critical size defect as an experimental model for craniomandibulofacial nonunions. Clin Orthop Relat Res. 1986; 205: 299-308.

29. Fearon J.A. A magnetic resonance imaging investigation of potential subclinical complications after in situ cranial bone graft harvestPlastic Reconstr Surg. 2000; 56: 1935-1939.

30. Jackson I.T., Helden G. and Marx R. Skull bone grafts in maxillofacial and craniofacial surgery. $\mathrm{J}$ Oral Maxillofac Surg. 1986; 44: 949-955.

31. Sagai M. and Bocci V. Mechanism of action involved in ozone therapy: Is healing induced via a mild oxidative stress? Med Gas Res. 2011; 20: 1-29.

32. Ozdemir H., Toker H., Balcı H. and Ozer H. Effect of ozone therapy on autogenous bone graft healing in calvarial defects: a histologic and histometric study in rats. J Periodont Res 2013; 48: 722-726. 